\title{
SiRNA in Vivo Delivery Systems: A New Frontier in Biotechnology
}

\section{Kathleen L Hefferon*}

Cell and Systems Biology, University of Toronto, 25 Willcocks St., Totonto, Ont. Canada

RNA interference can be defined as the process by which some RNAs undergo sequence-specific degradation, and results in reducing expression from transcripts which possess complementary sequence specificity. Small interfering RNAs (siRNAs) play a pivotal role in RNA interference, and are part of a series of cellular pathways, including a defense strategy against invading pathogens and a mechanism for the regulation of gene expression. The recent identification and elucidation of cellular RNA interference pathways has initiated a new discipline in molecular biology, and as a result, the advancement of siRNA technology as a powerful new tool by which to alter expression of specifically targeted genes. Although siRNA technology has rapidly moved forward from in vitro studies to the cell culture stage, a major challenge which remains is the development of effective in vivo siRNA delivery vehicles. The following editorial describes the current state of siRNA-based delivery technologies.

SiRNA comprises a class of double-stranded (ds)RNA molecules 20-25 nucleotides in length and containing 2-nucleotide 3' overhangs at each terminus. SiRNA's role in post-transcriptional gene silencing has been determined; these groups of molecules can prevent expression of genes which correspond to their corresponding specific sequence either by direct inhibition or destruction of the mRNA that they encode. This mechanism involves cleavage of the target RNA by a cellular endonuclease known as Dicer into short 21 bp fragments, which are then incorporated into a multiprotein complex known as the RISC (RNA induced silencing complex). The RISC selectively degrades one strand of the siRNA; the remaining strand of siRNA (the guide strand) becomes part of a siRNA/RISC complex, and is now capable of base pairing with and cleaving any complementary RNA species. As a result, RNAs derived from any organism which possess complementarity to the siRNA present in this complex are prevented from being used as a template for protein synthesis, and the gene corresponding to this particular RNA species becomes silenced.

SiRNA technology has been extensively utilized in reverse genomics studies to identify the function of an unknown gene in a biological pathway, to silence genes which play a specific role in diseases such as cancer, and even to change a crop phenotype or prevent an insect from acting as a vector for an infectious disease. While studies using cell culture have been paramount for the development of siRNA as a technology, effective delivery of siRNA into living organisms has proven to be the greatest challenge. A significant number of problems exist; among these are variations in siRNA stability in different cell types, toxicity of the delivery vehicle, immune response or adverse effects by the host, and the unintended co-suppression of additional, off-target sequences.

Today, efforts are being made to increase siRNA stability, decrease adverse effects and improve target cell uptake, and improve intracellular trafficking and release into the cytoplasm. One challenge that has been met with considerable success has been increasing the stability of siRNA and reducing its vulnerability to nucleases. Since siRNAs can be rapidly degraded by ribonucleases present in the blood, a number of chemical modifications have been introduced to their native structure which increases siRNA's in vivo stability, resistance to nucleases and retention in the body. In addition to this, the formation of siRNA conjugates with other stable compounds has also proven to be successful. Primary examples are siRNA-cholesterol conjugates, which have greatly improved the efficiency of siRNA uptake and internalization into cells. SiRNA has also been successfully conjugated to aptamers; for example, an aptamer specific for the HIV-1 envelope glycoprotein was demonstrated to deliver siRNAs to HIV infected cells, resulting in suppression of viral infection.

Lentivirus expression vectors derived from HIV have also been utilized as effective delivery vehicles for siRNA. Human immunodeficiency virus (HIV)-derived lentivectors have been engineered so that they can stably incorporate either siRNAs into the human body to permanently down-regulate expression of a particular gene, thus offering a potential gene therapy for cancers and other diseases. A measure of safety has been added to the use of lentivirus vectors by further engineering them to be replication-incompetent. To date, a lentiviral vector has been shown to silence green fluorescent protein (GFP) in mice and their offspring, proving its potential as a siRNA delivery vehicle. Other viral expression vectors, including adenovirus and herpes simplex virus type 1 , are under development as siRNA delivery systems. An advantage to the use of virus expression vectors for siRNA delivery is that they are able to provide tissue-specificity and high efficiency; however, issues such as unwanted immune responses by the host remain and can pose significant problems.

Nanocarriers, synthetic polymers a few hundred nanometers in size, are also under development as delivery vehicles for siRNAs. SiRNA nanocarriers have the advantage of being safe and can bind to and enter specific target cells efficiently, undergoing biodegradation and releasing their siRNA payload in a timely manner. To improve efficacy and minimize toxicity, lipid nanocarriers have been optimized with regard to lipid composition, siRNA-to-lipid ratio and particle size. For example, the polymer polyethylene glycol added to the outer surface of the nanoliposomes can increase their half-life as they circulate in the body. Alternatively, protamines, cationic polypeptides rich in arginine residues, can help to target siRNAs to specific tissue types including tumours. Antibodies have also been included on the outer surface of nanocarriers to selectively target nanoparticles to specific cell types.

Even more advanced innovations have led to the development of delivery vehicles which possess a single multifunctional polymer that can stably deliver the siRNA in the bloodstream, enable it to be efficiently recognized and taken up into the target cell, promote endosomal escape and trafficking into the cytoplasm, and still effectively

*Corresponding author: Kathleen L Hefferon, Cell and Systems Biology, University of Toronto, 25 Willcocks St., Totonto, Ont. Canada, Tel: (607) 387-6304; Fax: (607) 254-5454; E-mail: Kathleen.hefferon@alumni.utoronto.ca

Received August 26, 2012; Accepted September 05, 2012; Published September 09, 2012

Citation: Hefferon KL (2012) SiRNA in Vivo Delivery Systems: A New Frontier in Biotechnology. J Vaccines Vaccin 3:e109. doi:10.4172/2157-7560.1000e109

Copyright: (c) 2012 Hefferon KL. This is an open-access article distributed under the terms of the Creative Commons Attribution License, which permits unrestricted use, distribution, and reproduction in any medium, provided the original author and source are credited. 
Citation: Hefferon KL (2012) SiRNA in Vivo Delivery Systems: A New Frontier in Biotechnology. J Vaccines Vaccin 3:e109. doi:10.4172/2157$7560.1000 \mathrm{e} 109$

Page 2 of 2

silence the transcript of interest. Nanoparticle delivery systems such as these greatly reduce the dosage of siRNA required for gene silencing, lowering both cost and the potential for adverse effects from this course of treatment.

SiRNAs have been shown to play a role in a wide range of applications, from preventing difficult-to-treat human diseases to improving agronomic traits of crops. However, a number of stumbling blocks with regard to the widespread use of siRNA technologies for in vivo research remain to be resolved, including sensitivity to nuclease degradation, toxicity to the host, short retention time, inefficient uptake into target cells and poor release into the cytosol. The current task is to implement new innovations, a few of which have been mentioned in this editorial, to resolve these problems and make siRNA technology a viable innovation for the future. 